\title{
Evaluation of contact sensitivity to food additives in children with atopic dermatitis
}

\author{
Hülya Anıl, Koray Harmancı \\ Department of Paediatric Allergy and Immunology, Faculty of Medicine, Osmangazi University, Eskisehir, Turkey \\ Adv Dermatol Allergol 2020; XXXVII (3): 390-395 \\ DOI: https://doi.org/10.5114/ada.2020.96112
}

\begin{abstract}
Introduction: Atopic dermatitis (AD) is a chronic inflammatory disease caused by the complex interaction of genetic, immune and environmental factors such as food and airborne allergens. The atopy patch test (APT) is a useful way to determine delayed-type hypersensitivity reactions to food and aeroallergens. Many studies have also suggested that food additives are associated with dermatologic adverse reactions and the aggravation of pre-existing atopic dermatitis symptoms.

Aim: To elucidate the contact sensitivity to food additives in children suffering from AD by using standardized atopy patch testing.

Material and methods: A total of 45 children with AD and 20 healthy children have been enrolled. All the children have regularly consumed food containing additives, and were subjected to atopy patch tests.

Results: In total, 28 (62\%) children with AD and $4(20 \%)$ healthy children have had positive patch test reactions to $\geq 1$ allergens. There has been a significant difference $(p=0.04)$ between the groups in terms of the positivity rate in the patch test and the most common allergen that elicited positive patch test results in the AD group was azorubine ( $n=11,24.4 \%, p=0.014)$.

Conclusions: In our study, contact sensitivity was detected more frequently in AD patients. Food additives may play a role in the development and exacerbation of AD. Atopy patch testing with food additives can be useful in the treatment and follow-up of children with AD.
\end{abstract}

Key words: atopic dermatitis, food additives, patch test, children, azorubine.

\section{Introduction}

Atopic dermatitis (AD) is a chronic inflammatory skin disease characterized by pruritic and eczematous skin lesions [1]. A number of environmental factors have been implicated in the pathogenesis of AD. Food allergens (mostly in children) and aeroallergens are the most relevant allergens in $\mathrm{AD}[2]$. In the chronic inflammatory process of AD, type 1 and type 4 hypersensitivity reactions to allergens may be responsible. Elevated serum Immunoglobulin E (IgE) levels can be demonstrated in $80 \%$ to $85 \%$ of patients with $A D$, and a similar number have immediate skin test response or positive in vitro tests to food and inhalant allergens. The relationship between the course of $A D$ and implicated allergens has been difficult to establish [3].

Food additives are extensively used in the food industry and include thousands of natural and synthetic substances used as flavourings, colorants, preservatives, sweeteners, antioxidants and thickeners [4]. "Food additive" means any substance not normally consumed as a food by itself and not normally used as a typical ingredient of the food, whether or not it has nutritive value, the intentional addition of which to food for a technological purpose in the manufacture, processing, preparation, treatment, packing, packaging, transport or holding of such food results, or may be reasonably expected to result, in it or its by-products becoming a component of or otherwise affecting the characteristic of such foods [5]. Food additives are associated with dermatologic adverse reactions, such as urticaria, angioedema and the aggravation of atopic dermatitis symptoms [6]. Atopy patch tests (APT) which are devoid of any side-effects have been introduced as part of the diagnostic work-up for the diagnosis of non-IgE mediated allergy, seen in conditions like AD [7]. However a few studies have investigated the atopy patch test for food additives in patients with $A D$.

Address for correspondence: Hülya Anıl, Department of Paediatric Allergy and Immunology, Eskisehir Osmangazi University Hospital, Meselik-Eskisehir, Turkey, phone: +90 222 2397929, fax: +90 222 2397440, e-mail: hulyaanil26@gmail.com Received: 2.12.2018, accepted: 20.01.2019. 


\section{Aim}

The present study is aimed to elucidate the contact sensitivity to food additives and determine if food additives play a role in the aetiology of AD.

\section{Material and methods}

The study participants have comprised of 65 children whose age range has been between 2 and 18. All the children have been followed prospectively and clinically evaluated by the same allergist. The definition of atopic dermatitis has been made with reference to Hanifin and Rajka [8]. Inclusion criteria for the test have been regular consumption of foods containing additives (e.g., chocolate, chips, ice cream, fruit juice, gumdrops, candy, biscuit, baked goods, fruit yoghurt, pudding, jam, syrup, meat products, ketchup and chewing gum).

Patients with systemic, acute infectious, autoimmune and other allergic diseases have been excluded from the study. Children have not taken any oral immunosuppressive drugs including oral corticosteroids and phototherapy for at least 1 month before the test. Oral antihistamine drugs and topical treatment of the back with corticosteroids have been discontinued for at least 7 days before the test. Twenty healthy individuals with no personal or family history of asthma and other atopic diseases have been selected as the control group. All children enrolled have completed a questionnaire designed to observe the presence of a food additive-related hypersensitivity and have undergone the patch test. Both groups have responded to a questionnaire evaluating the frequency of consumption of chocolate, chips, ice cream, fruit juice, gumdrops, candy, biscuit, baked goods, fruit yoghurt, pudding, jam, syrup, meat products (salami and sausages), ketchup and chewing gum.

Leukocyte, neutrophil, lymphocyte, eosinophil count measurements have been performed within approximately 60 min after blood sampling with Coulter Hmx Hematology Analyzer (Beckman Coulter, Inc., CA, USA) with the use of original method and reagents. The ratios of blood cells have been calculated by dividing the percentage of cells in complete blood count analysis. Skin prick testing has been carried out using a panel of standardized allergens (Stallergenes SA, Antony, France) including cow's milk, egg yolk, wheat, nuts and soya. A positive prick test response has been defined as a wheal diameter of $3 \mathrm{~mm}$ or larger to at least one of these allergens. Total serum IgE has been determined by ImmunoCAP (Phadia, Uppsala, Sweden).

Atopy patch tests (APT) have been performed pursuant to the European Task Force on Atopic Dermatitis (ETFAD) on the non-lesional skin. The occlusion time has been $48 \mathrm{~h}$ and the patch test have been read at 48 and $72 \mathrm{~h}$. The APT results have been graded according to ETFAD standards: no reaction or erythema without infiltration (-), erythema and infiltration $(+)$, erythema and few papules $(++)$, erythe- ma and many or spreading papules (+++), erythema, papules and vesicles (++++) [9]. Atopy patch testing has been performed using $8 \mathrm{~mm}$ patch test chambers (AllergEAZE, Smart Practice, Phoenix, AZ) with food additive series (Brial Allergen, Greven, Germany) including aspartame, azorubine, amaranth, benzoic acid, butylhydroxyanisole, brilliant black, butylhydroxytoluene, cochineal red (ponceau 4R), carmine, erythrosine-b, formic acid, patent blue, pectin, quinoline yellow, sorbic acid, monosodium glutamate, sodium diphosphate, sodium disulphite, sulfur dioxide, saccharine, sodium formiate, sodium alginate, and tartrazine. In our study, SCORAD calculation and the readouts of skin and atopy patch tests have been performed by a single expert (the author) in order to minimise manual errors.

E numbers are number codes for food additives and are usually found on food labels throughout the European Union. The 'E' stands for Europe or European Union. Normally, each food additive is assigned a unique number, and though occasionally, related additives are given an extension to another E-number. The concentrations of food additives, vehicles and E numbers used for allergens are given in Table 1.

This study has been approved by the institutional review board of the Faculty of Medicine. Informed consent has been obtained from parents of each patient before his or her inclusion in the study. The study has been conducted in compliance with the Declaration of Helsinki.

\section{Statistical analysis}

Statistical analyses have been performed via SPSS statistical software (version 21.0; Armonk, NY, USA). Continuous variables are presented as mean \pm SD and median (range). Categorical variables are shown as frequency and percentage. Student $t$ tests, Pearson $\chi^{2}$ analysis, and Mann-Whitney U-test have been used to compare differences between two specific groups, and Spearman rank correlation coefficients have been assessed as the measures of correlation between variables of interest. The level of statistical significance has been set as $p=0.05$.

\section{Results}

Forty five patients (28 girls, 17 boys) within the age range of 24 months to 17 years (median age: 7 years) with $A D$ and 20 healthy children (12 girls and 8 boys) within the age range of 36 months to 17 years (median age: 8 years) have been included in this study. The age and gender distributions of the patient and control groups have been similar (Table 2). White blood cell count, eosinophil count and the percentage of eosinophil in hemogram have been significantly higher in the $A D$ group than those of the control group.

According to the SCORAD index, $80.2 \%$ of AD patients have had mild disease while $19.8 \%$ have had moderate to severe disease. Age, gender, eosinophil count and serum total IgE levels have not differed significantly between the 
Table 1. Concentrations of food additives, vehicles and code for allergens

\begin{tabular}{|c|c|c|c|}
\hline Allergen & Concentration \% & Vehicle & Code \\
\hline Aspartame & 0.1 & Coca & E-951 \\
\hline Azorubine & 0.1 & $\mathrm{EtOH}$ & $\mathrm{E}-122$ \\
\hline Amaranth & 0.1 & Coca/glyc & E-123 \\
\hline Benzoic acid & 1 & EtOH/glyc & E-210 \\
\hline Butylhydroxyanisole & 2 & $\mathrm{EtOH}$ & E-320 \\
\hline Brilliant black & 0.1 & Coca/glyc & E-151 \\
\hline Butylhydroxytoluene & 1 & $\mathrm{EtOH}$ & E-321 \\
\hline Cochineal red & 1 & Coca/glyc & E-124 \\
\hline Carmine & 0.5 & Coca/glyc & E-120 \\
\hline Erythrosine-B & 0.25 & $\mathrm{EtOH} /$ glyc & E-127 \\
\hline Formic acid & 1 & Water & E-236 \\
\hline Patent blue & 0.25 & Coca/glyc & E-131 \\
\hline Pectin & 1 & Coca & E-440 \\
\hline Quinoline yellow & 0.1 & Coca/glyc & E-104 \\
\hline Sorbic acid & 2 & EtOH/glyc & E-200 \\
\hline Sodium glutamate & 1 & Coca & E-621 \\
\hline Sodium diphosphate & 1 & Coca/glyc & E-450 \\
\hline Saccharine & 0.1 & Coca/glyc & E-954 \\
\hline Sodium disulphite & 1 & Petrolatum & $E-221$ \\
\hline Sodium nitrite & 2 & Wat/glyc & E-250 \\
\hline Sodium formiate & 2 & Coca/glyc & E-237 \\
\hline Sodium alginate & 1 & Coca & E-401 \\
\hline Tartrazine & 1 & Coca/glyc & E-102 \\
\hline
\end{tabular}

Table 2. Demographic and laboratory characteristics of the children

\begin{tabular}{|c|c|c|c|}
\hline Parameter & $\begin{array}{c}\text { Atopic } \\
\text { dermatitis } \\
(n=45)\end{array}$ & $\begin{array}{l}\text { Control } \\
(n=20)\end{array}$ & $P$-value \\
\hline Gender (F/M) & $28 / 17$ & $12 / 8$ & $>0.05$ \\
\hline Age [year] mean \pm SD & $7.44 \pm 4.19$ & $8.15 \pm 4.45$ & $>0.05$ \\
\hline $\begin{array}{l}\text { White blood cell count } \\
{\left[\times 10^{3} / \mu \mathrm{l}\right]}\end{array}$ & $8326 \pm 2685$ & $6955 \pm 1124$ & 0.032 \\
\hline Neutrophil count $\left[\times 10^{3} / \mu \mathrm{l}\right]$ & $4097 \pm 2078$ & $3335 \pm 1143$ & $>0.05$ \\
\hline Lymphocyte count $\left[\times 10^{3} / \mu \mathrm{l}\right]$ & $3208 \pm 1429$ & $2845 \pm 934$ & $>0.05$ \\
\hline \multicolumn{4}{|l|}{ Ratio of neutrophil: } \\
\hline Lymphocyte (N/L) & $1.55 \pm 1.24$ & $1.38 \pm 0.88$ & $>0.05$ \\
\hline Percentage of eosinophil (\%) & $3.1 \pm 2.55$ & $1.92 \pm 1.63$ & 0.046 \\
\hline Eosinophil count $\left[\times 10^{3} / \mu \mathrm{l}\right]$ & $262 \pm 21$ & $140 \pm 127$ & 0.015 \\
\hline Serum total IgE [IU/ml] & $399 \pm 791$ & $68.2 \pm 100$ & $>0.05$ \\
\hline
\end{tabular}

mild $A D$ and the moderate to severe AD group. Ten (22\%) patients in the AD group have had positive skin prick test results, $40 \%$ of which were positive to hen's egg, $40 \%$ to cow's milk, and $20 \%$ to both. But none of them have had any food allergy history. None of the individuals in the control group have shown sensitization to SPT.

Sixty (92.3\%) children have consumed an average of three kinds of snacks containing food additives daily. Although the consumption of gumdrops, sausages and salami were comparable between the AD and control groups, consumption of fruit yoghurt, ice cream, jam, and cake was significantly higher in the AD group $(p=0.005)$.

Atopy patch tests have been performed for all the children, $62 \%(n=28)$ of the AD group have had positive APT results. The APT results for food additives are shown in Table 3. The most common allergen to yield positive APT results has been azorubine ( $n=11,24.4 \%)$. Twentyeight of 45 patients have had positive APT results, meaning that they are allergic to 23 different food additives; 9 of these positive reactions have been $3+, 28$ have been $2+$, and the remaining 8 have been $1+$. In the control group, 4 of 20 children have had positive APT results to five different food additives; all of the positive reactions have been 1+. Compared with the control group, the AD group have had higher positive APT results for azorubine (24.4\% and $0 \%$, respectively, $p=0.014)$. In the AD group 11 patients have had positive APT results for azorubine $(n=3,3+; n=5,2+; n=3,1+)$.

There has been no significant difference in terms of age, gender, SCORAD index, the presence of eosinophilia, serum total IgE levels, neutrophil count, lymphocyte count, ratio of neutrophil lymphocyte between the APTpositive and negative patients with AD.

\section{Discussion}

$A D$ is an inflammatory disease of the skin. Patients with AD have been observed to have a neutrophilic inflammation with eosinophilic inflammation [10-12]. In our study, the mean white blood cell, eosinophil count and the percentage of eosinophil have been significantly higher in the AD group than those of the control group. The mean neutrophil and neutrophil-lymphocyte ratios (NLR) in children with $A D$ have not been statistically significant compared to those of the control group. Although $A D$ is an inflammatory skin disease and neutrophil and NLR are markers of inflammation, the reason of the nonsignificant neutrophil and NLR values between the groups may be due to the small number of patients and having mostly mild to moderate AD. The number of eosinophils in $A D$ may be higher than the normal values [13].

Although food additives have been used since ancient times, allergic reactions to additives have been reported in very few population-based studies and case reports. The prevalence of reactions to food additives is estimated at $1-2 \%$ in unselected populations of healthy 
children, and higher in atopic children, ranging from $7 \%$ to $2 \%[14,15]$.

Studies document the association of food additives with adverse skin reactions, such as urticaria, angioedema, and aggravation of pre-existing AD; however, the evidence of a causal relationship is discrepant [16]. Ehlers et al. [17] have confirmed the role of hypersensitivity to certain food additives (including, azo and nonazo food) in children with chronic urticaria. Of 16 children with moderate to severe chronic urticaria, 13 (81\%) have entered a remission period under a stringently controlled low-pseudoallergen diet, and all have reacted with wheals later upon discontinuation of their diet. They have reported that in children with chronic urticaria food additives play a more important role in eliciting hypersensitivity reactions than in adult patients. Studies carried out with both children and adults have shown a discrepancy between the perception of patients and the reported prevalence of reactions to food additives. Rajan et al. [18] have assessed hypersensitivity to food additives based on the challenge testing in patients with chronic idiopathic urticaria. Among the 100 patients, only 2 have had a positive urticarial response on single blind challenge to 11 common food additives.

APTs show a higher specificity than skin prick and specific IgE tests since the pathophysiological mechanism of the reaction induced is very similar to that which occurs in AD lesions. We have found positive APT to food additives in $62 \%$ of children with AD. APT positivity has been significantly more common in patients with $A D$ than that of healthy groups $(p=0.004)$. In the AD group, 28 of 45 patients have had 45 positive APT results to 23 different food additives, and 4 of 20 children have had positive APT results to five different food additives. All of the positive reactions have been $1+$ in the control group. These findings suggest that atopy patch testing in individuals with $A D$ is more specific than in those without and food additives other than carmine did not seem to be associated with AD.

Until now, a few controlled studies about the food additives role in AD have revealed similar results. Park et al. [6] have shown positive APT to food additives in $5.6 \%$ of patients with allergy, and this lower result could be related to fewer types of food additives (7 vs. 23 in our study). Since there is a possibility that false positive APT occurs to additives due to irritation, control APT has been conducted on non-allergic individuals in our study. The absence of strong and much positive APT results in the control group supports the effectiveness of APT in diagnosis of food allergy in children with AD.

Paediatric AD patients may have an increased risk of allergen penetration through the skin, and most allergies are initiated in childhood. Therefore, we believe that food additives could worsen AD, especially in paediatric patients but the selection of the standard test for discriminating hypersensitivity to food additives is still
Table 3. The most common patch test results and the associated allergens

\begin{tabular}{lccc}
\hline Parameter & $\begin{array}{c}\text { Atopic dermatitis } \\
(n=45)\end{array}$ & $\begin{array}{c}\text { Control } \\
(n=20)\end{array}$ & $P$-value \\
\hline $\begin{array}{l}\text { Number of individuals } \\
\text { with positive reactions }\end{array}$ & $28(62)$ & $4(20)$ & 0.004 \\
\hline Azorubine, $n(\%)$ & $11(24.4)$ & $0(0)$ & 0.014 \\
\hline Formic acid, $n(\%)$ & $7(15.6)$ & $3(0)$ & $>0.05$ \\
\hline Carmine, $n(\%)$ & $6(13.3)$ & $1(0)$ & $>0.05$ \\
\hline Cochineal red, $n(\%)$ & $6(13.3)$ & $1(0)$ & $>0.05$ \\
\hline Amaranth, $n(\%)$ & $6(13.3)$ & $1(0)$ & $>0.05$ \\
\hline
\end{tabular}

under investigation. The APT is a useful tool for determining delayed-type hypersensitivity reactions to foods and aeroallergens in AD [19].

In a recent study, Çatli et al. [20] have investigated the role of food additives sensitivity based on atopy patch tests in AD children. They have reported that forty one percent of the atopic dermatitis group $(n=14)$ and $15.2 \%(n=5)$ of the healthy children have had positive APT results to food additives $(p=0.036)$. They have also reported the following positive reactions: cochineal red in 8 patients; carmine in 6 patients; sodium nitrite in 4 patients; pectin, erythrosine and benzoic acid in 3 patients; sorbic acid, sodium disulphide, and aspartame in 2 patients; patent blue, quinolone yellow, sodium glutamate, sodium alginate, and tartrazine in 1 patient. Among their patients, positive APT results for carmine have been significantly higher in the $A D$ than those of the control group, whereas the differences for other food additives have not been statistically significant. In our study, $62 \%$ of the patients have had positive reactions, and $24.4 \%$ of them have been azorubine. In contrast to Çatlı et al., most of the present study's atopic children have had a positive reaction to azorubine and not to carmine. They have indicated that food additives hypersensitivity should be considered as an additional precipitating factor in children with AD.

Azo dyes are a large class of synthetic organic dyes some of which are used as food colorants. Azorubine is mostly used in ice cream, yoghurt, soft drinks, instant puddings, flavoured chips, cake mixes, custard, candy, and fermented dairy products. They are also used in some pharmaceutical products, cosmetics, moisturizers and crayons [21]. There are reports showing that they may cause attention deficit and hyperactivity disorder in children and teenagers [22]. In the literature, there is no study or case report about the relationship between azorubine and AD. Gülseren et al. [23] have studied the association between food additives and recurrent aphthous stomatitis. They have reported that 11 of 21 patients have had a positive APT to azorubine.

In addition, Amin et al. [24] have detected altered kidney and liver function and oxidative stress biomarkers after tartrazine and azorubine intake in male rats. 
According to their data, in the treated group the levels of alanine aminotransferase, aspartate aminotransferase, total protein, and albumin have been significantly increased in comparison to those of the control group, especially at tenfold concentration exposure. Similarly, in liver homogenates, the level of glutathione, superoxide dismutase, and catalase have decreased, while malondialdehyde levels have increased at tartrazine and azorubine and as a result they possibly induce oxidative stress $[24,25]$. Increased tissue concentrations of enzymes involved in the oxidative mechanisms indicate that these food colorants may intervene in the multi-step process of inflammation.

This study's patch test findings suggest that food additives and type 4 hypersensitivity play a significant role as a precipitating factor of $A D$ in some individuals. The present study has investigated the role of food additives in AD based only on patch testing; however, skin prick testing in conjunction with anamnesis and physical examination, and diagnostic skin testing are the cornerstone for evaluating food allergy and investigating type 1 hypersensitivity involvement [26].

The high ratio of positive APT reactions to azorubine and consumption of fruit yoghurt, ice cream, jam, and cake significantly higher in patients with $A D$ indicates that azorubine may play a role in AD. Although the double-blind placebo-controlled challenge test is the criterion standard method for diagnosis of food allergy. Our study has been limited primarily by the lack of oral challenge in all patients with allergy and the control group. This may lead to less precise estimation of hypersensitivity to food additives. This could have been due to practical troubles in performing challenge with various kinds of food additives in all studied individuals. Also, there are no standardized protocols with food additives in the literature.

\section{Conclusions}

In the present study, about $62 \%$ of AD children with a positive reaction to food additives showed positive APT, it may be considered the potential utility of APT to identify the role of food additives in children with AD. This study also showed that it might be useful to perform APT with azorubine in children with atopic dermatitis who report having had symptoms after eating foods containing azorubine. Further larger and well-controlled observational studies are required in the future to consolidate the association between food additives and AD.

\section{Acknowledgments}

The work was performed at the Eskişehir Osmangazi University.

\section{Conflict of interest}

The authors declare no conflict of interest.

\section{References}

1. Addor FA, Takaoka R, Rivitti EA, et al. Atopic dermatitis: correlation between non-damaged skin barrier function and disease activity. Int J Dermatol 2012; 51: 672-6.

2. Turjanmaa K, Darsow U, Niggemann B, et al. EAACI/GA2LEN position paper: present status of the atopy patch test. Allergy 2006; 61: 1377-84.

3. Caubet JC, Eigenmann PA. Allergic triggers in atopic dermatitis. Immunol Allergy Clin North Am 2010; 30: 289-307.

4. Skypala IJ, Williams M, Reeves L, et al. Sensitivity to food additives, vaso-active amines and salicylates:a review of the evidence. Clin Transl Allergy 2015; 5: 34.

5. World Health Organization (WHO/Food and Agriculture Organization of the United Nations (FAO] (2013] Codex Alimentarius Commission, procedural manual. 21st edition. WHO/ FAO, Rome.

6. Park HW, Park CH, Park SH, et al. Dermatologic adverse reactions to 7 common food additives in patients with allergic diseases: a double-blind, placebo-controlled study. J Allergy Clin Immunol 2008; 121: 1059-61.

7. Yang H, Xiao YZ, Luo XY, et al. Diagnostic accuracy of atopy patch tests for food allergy in children with atopic dermatitis aged less than two years. Allergol Immunopathol 2014; 42: 22-8.

8. Hanifin JM, Rajka G. Diagnostic features of atopic dermatitis. Acta Derm Venereol Suppl 1980; 92: 44-7.

9. Darsow U, Wollenberg A, Simon D, et al. ETFAD/EADV eczema task force 2009 position paper on diagnosis and treatment of atopic dermatitis. J Eur Acad Dermatol Venereol 2010; 24: 317-28.

10. Choy DF, Hsu DK, Seshasayee D, et al. Comparative tanscriptomic analyses of atopic dermatitis and psoriasis reveal shared neutrophilic inflammation. J Allergy Clin Immunol 2012; 130: 1335-43.

11. Spergel JM, Mizoguchi E, Brewer JP, et al. Epicutaneous sensitization with protein antigen induces localized allergic dermatitis and hyperresponsiveness to methacholine after single exposure to aerosolized antigen in mice. J Clin Invest 1998; 101: 1614-22.

12. Hon KL, Wang SS, Pong NH, et al. Circulating immunoglobulins, leucocytes and complements in childhood-onset atopic eczema. Indian J Pediatr 2013; 80: 128-31.

13. Dogru M, Citli R. The neutrophil-lymphocyte ratio in children with atopic dermatitis: a case-control study. Clin Ter 2017; 168: 262-5.

14. Fuglsang G, Madsen C, Saval P, et al. Prevalence of intolerance to food additives among Danish school children. Pediatr Allergy Immunol 1993; 4: 123-9.

15. Fuglsang G, Madsen G, Halken S, et al. Adverse reactions to food additives in children with atopic symptoms. Allergy 1994; 49: 31-7.

16. Wilson BG, Bahna SL. Adverse reactions to food additives. Ann Allergy Asthma Immunol 2005; 95: 499-507.

17. Ehlers I, Niggemann B, Binder C, Zuberbier T. Role of nonallergic hypersensitivity reactions in children with chronic urticaria. Allergy 1998; 53: 1074-7. 
18. Rajan JP, Simon RA, Bosso JV. Prevalence of sensitivity to food and drug additives in patients with chronic urticaria. J Allergy Clin Immunol Pract 2014; 2: 168-71.

19. Edwards KP, Martinez BA. Atopy patch testing for foods: a review of the literature. Allergy Asthma Proc 2014; 35: 435-43.

20. Çatli G, Bostanci I, Ozmen S. Is patch testing with food additives useful in children with atopic eczema? Pediatric Dermatology 2015; 32: 684-9.

21. Elbanna K, Sarhan OM, Khider M, et al. Microbiological, histological, and biochemical evidence for the adverse effects of food azo dyes on rats. Food Drug Anal 2017; 25: 667-80.

22. McCann D, Barrett A, Cooper A. Food additives and hyperactive behaviour in 3-year-old and 8/9-year-old children in the community: a randomised, double-blinded, placebocontrolled trial. Lancet 2007; 370: 1560-7.

23. Gülseren D, Hapa A, Ersoy-Evans S, et al. Is there a role of food additives in recurrent apthous stomatitis? A prospective study with patch testing. Int I Dermatol 2017; 56: 302-6.

24. Amin KA, Abdel Hameid HH, Abd Elsttar AH. Effect of food azo dyes tartrazine and carmoisine on biochemical parameters related to renal, hepatic function and oxidative stress biomarkers in young male rats. Food Chem Toxicol 2010; 48: 2994-9.

25. Tsuda S, Murakami M, Matsusaka N, et al. DNA damage induced by red food dyes orally administered to pregnant and male mice. Toxicol Sci 2001; 61: 92-9.

26. Wardhana, Datau EA. Recurrent aphthous stomatitis caused by food allergy. Acta Med Indones 2010; 42: 236-40. 